Fausto Aloísio Pedrosa Pimenta (iD) https://orcid.org/0000-0002-9169-0443

Rafaella Lemos Alves ${ }^{\text {b }}$

(iD) https://orcid.org/0000-0002-7387-8936

Fernando Luiz Pereira de Oliveira ${ }^{c}$

(D) https://orcid.org/0000-0001-6513-3339

Raimundo Marques do Nascimento Neto a

(iD) https://orcid.org/0000-0003-2038-5203

George Luiz Lins Machado Coelho ${ }^{d}$

(iD) https://orcid.org/0000-0002-9806-9721

Silvia Nascimento de Freitas ${ }^{b}$

(iD) https://orcid.org/0000-0002-4119-0352

a Universidade Federal de Ouro Preto (UFOP), Departamento de Clínicas Pediátrica e do Adulto. Ouro Preto, MG, Brasil.

b Universidade Federal de Ouro Preto (UFOP), Escola de Nutrição. Ouro Preto, MG, Brasil.

${ }^{\mathrm{C}}$ Universidade Federal de Ouro Preto (UFOP), Departamento de Estatística. Ouro Preto, MG, Brasil.

¿Universidade Federal de Ouro Preto (UFOP), Departamento de Medicina de Família, Saúde Mental e Saúde

Coletiva. Ouro Preto, MG, Brasil.

Contato:

Fausto Aloísio Pedrosa Pimenta

E-mail:

fpimenta@ufop.edu.br

Trabalho baseado em dissertação de mestrado de Rafaella Lemos Alves, intitulada Qualidade de vida e excesso de peso de trabalhadores de turnos alternantes da região dos inconfidentes, MG, defendida em 2014 na

Universidade Federal de Ouro Preto.

O trabalho recebeu auxílio financeiro do Conselho Nacional de Desenvolvimento Científico e Tecnológico $(\mathrm{CNPq})$, projetos $\mathrm{PQ}$ 305999/2014-1 e PQ 300825/2016-1, da Fundação de Amparo à Pesquisa do Estado de Minas Gerais (Fapemig), e da Coordenação de Aperfeiçoamento de Pessoal de Nível Superior (Capes), Projeto Prevenção da Fadiga ( $\left.\mathrm{n}^{\circ} 1325\right)$, Laboratório de Cardiometabolismo.

Os autores declaram que não há conflitos de interesses e que o trabalho não foi apresentado em eventos científicos.

\section{Qualidade de vida e excesso de peso em trabalhadores em turnos alternantes}

\author{
Quality of life and overweight in alternating shift workers
}

\section{Resumo}

Objetivo: identificar a percepção dos trabalhadores de turnos alternantes de uma mineradora da região dos Inconfidentes, Minas Gerais, sobre sua qualidade de vida (QV) e analisar sua associação com indicadores de excesso de adiposidade corporal. Métodos: estudo transversal com 437 trabalhadores em turnos alternantes com um ou mais fatores de risco cardiovascular. Dados sociodemográficos e clínicos foram analisados. A QV foi avaliada com o questionário SF-36 e a adiposidade corporal estimada a partir das medidas antropométricas e de composição corporal. Resultados: a pontuação para os domínios da QV variou de 67 a 100, no entanto, o aumento de gordura corporal apresentou correlação negativa com os domínios saúde geral, vitalidade e capacidade funcional. Foi observada, por análise de cluster, a formação de dois agrupamentos, um composto pelos domínios da QV e o outro constituído pelos indicadores de composição corporal. Não foi identificada associação entre os escores estimados de QV dos trabalhadores de turno e o tempo de trabalho. Conclusão: o declínio da QV apresentou associação com o excesso de adiposidade corporal. Recomenda-se a adoção de medidas visando reduzir o excesso de adiposidade corporal e melhorar a qualidade de vida dos trabalhadores em turno alternante da mineração.

Palavras-chave: qualidade de vida; saúde do trabalhador; estado nutricional; trabalho em turnos; mineração.

\begin{abstract}
Objective: to identify the perception that alternating shift workers from a mining company in the region of Inconfidentes, Minas Gerais, Brazil, have about their quality of life (QoL) and to analyse its association with indicators of excess body adiposity. Methods: cross-sectional study involving 437 alternating shift workers with one or more cardiovascular risk factors. Sociodemographic and clinical data were analyzed. QoL was evaluated by the SF-36 questionnaire. Body adiposity was estimated through anthropometric and body composition measurements. Results: the scores for QoL domains ranged from 67 to 100 , however, body fat increasing showed a negative correlation with general health, vitality and functional capacity domains. Through cluster analysis, the authors observed the formation of two groups, one comprising the QoL domains, and a second made up of body composition indicators. No association was found between the shift workers estimated QoL scores and their shift working lifetime. Conclusion: the QoL decline was associated with body adiposity excess. The recommendation is the adoption of measures aimed at reducing excess body adiposity and improving mining alternating shift workers' quality of life.
\end{abstract}

Keywords: quality of life; occupational health; nutritional status; shift work; mining. 


\section{Introdução}

A Organização Mundial de Saúde (OMS) ${ }^{1}$ define qualidade de vida (QV) como a percepção que o indivíduo tem de sua posição na vida, no contexto da cultura e sistema de valores nos quais vive, e em relação aos seus objetivos, expectativas, padrões e preocupações. A avaliação da QV em grupos populacionais é importante para verificar fatores intervenientes em seus domínios físico e mental. Também poderá servir para comparar a utilização de novos insumos e tecnologias pelos indivíduos, visando avaliar seu impacto na vida das pessoas.

Embora autores como Sampaio ${ }^{2}$ proponham que a QV no trabalho seja um guarda-chuva teórico que repousa em três conceitos nucleares - o humanismo, que ora repousa em motivação, ora em satisfação; a participação do empregado em decisões de gestão; e o bem-estar -, entende-se que os aspectos que envolvem a QV do trabalhador transcendem o próprio trabalho. Estes incluem também fatores individuais (saúde e comportamento) e sociais. Portanto, os instrumentos que avaliam a QV devem contemplar todos estes aspectos.

Assim, o trabalho desempenhado, junto com as condições físicas, mentais, emocionais, econômicas, ambientais e sociais, pode interferir na QV do indivíduo. Entre as condições físicas, o excesso de peso e a distribuição da gordura corporal são fatores associados a uma ampla gama de complicações e agravos à saúde, os quais podem reduzir a $\mathrm{QV}^{3,4}$.

Estudos têm demonstrado que trabalhadores de serviços noturnos que exercem suas atividades em sistemas de turno apresentam índice de massa corporal (IMC) maior em comparação àqueles cujo horário de trabalho é no período diurno ${ }^{5,6}$. Essa alteração pode ser decorrente das adaptações do organismo a essa rotina de trabalho, as quais acarretam mudanças no metabolismo e no comportamento alimentar ${ }^{7}$.

Morgan et al. ${ }^{8}$, avaliando 110 homens trabalhadores em turnos, encontraram uma média de IMC de $30,5 \mathrm{~kg} / \mathrm{m}^{2}$ e circunferência da cintura (CC) de 100,7 cm. Pepłońska et al. ${ }^{9}$ acompanharam trabalhadores submetidos a esta escala por 14 anos, e observaram um aumento de até 10\% no IMC em trabalhadores poloneses. No Brasil, Codarin et al. ${ }^{10}$, avaliando 470 motoristas de caminhão que realizavam a maior parte de suas atividades no período noturno, verificaram que $62,4 \%$ apresentavam IMC superior a $25 \mathrm{~kg} / \mathrm{m}^{2}$. Battaus e Monteiro ${ }^{11}$ identificaram 47,8\% homens, trabalhadores com diferentes funções de uma metalúrgica, com IMC superior a $25 \mathrm{~kg} / \mathrm{m}^{2}$.

A acumulação de tecido adiposo está associada à diminuição da capacidade funcional e à redução da QV. Alguns estudos demonstram elevada prevalência de excesso de adiposidade entre trabalhadores de turno em diferentes regiões do mundo, o que pode ser decorrente das alterações metabólicas ocasionadas pela dessincronização do ciclo circadiano, podendo afetar a produtividade e aumentar o risco de absenteísmo por doenças e acidentes ${ }^{8-11}$. No entanto, no Brasil, a maioria dos estudos realizados com trabalhadores de turno tomam como população-alvo profissionais da área de saúde e motoristas de transportadoras ${ }^{9,10}$. São poucos os que analisam a população de trabalhador de turno alternante da mineração e o efeito do excesso de adiposidade corporal na QV desses indivíduos. Por outro lado, grande parte dos estudos utiliza o IMC como indicador de excesso de adiposidade corporal. Desta forma, para estabelecer ações de saúde há uma clara necessidade de estudos epidemiológicos que demonstrem se a QV é influenciada pelo excesso e distribuição da adiposidade corporal em trabalhadores de turnos alternantes e quais domínios da QV podem ser afetados pelo excesso de adiposidade corporal.

Assim, este estudo teve por objetivo identificar a percepção dos trabalhadores de turnos alternantes de uma mineradora da região dos Inconfidentes, Minas Gerais, sobre sua qualidade de vida (QV) e analisar sua associação com indicadores de excesso de adiposidade corporal.

\section{Métodos}

Estudo com delineamento transversal descritivo, conduzido em empresa mineradora de grande porte situada na região dos Inconfidentes, Minas Gerais, em 2012.

A população do estudo foi selecionada entre os 952 trabalhadores que participaram da primeira etapa do Projeto Prevenção da Fadiga em Trabalhadores da Mineração do Estado de Minas Gerais, desenvolvido pela Universidade Federal de Ouro Preto (UFOP). Inicialmente, foram convidados a participar deste estudo específico os que exerciam função administrativa e os que atuavam na função de motorista de caminhão fora de estrada (caminhões de grande porte, com alta capacidade de transporte de carga pesada, projetados para uso em áreas de mineração).

Após esclarecimentos sobre o estudo serem oferecidos pelos pesquisadores e a assinatura do termo de consentimento livre e esclarecido (TCLE) pelos trabalhadores, foi realizada triagem visando identificar os indivíduos que possuíam um ou mais fatores de risco para doença cardiovascular. Essa etapa 
envolveu avaliação clínica e nutricional e aplicação de instrumento de avaliação de QV.

Durante o processo houve perdas devido a recusas, férias, folgas e demissões; optou-se por excluir os trabalhadores com função administrativa, sendo definidos como participantes apenas os motoristas de caminhão fora de estrada. Após as avaliações, foram excluídos os trabalhadores que não possuíam fator de risco para doença cardiovascular e os que possuíam informações incompletas referentes à QV, resultando nos 437 participantes deste estudo.

A jornada de trabalho destes indivíduos era de turno alternante, com 6 horas por turno e descanso de 12 horas entre turnos. A alternância dos horários de trabalho e de descanso se dava de forma rotativa. O turno inicial se dava das $19 \mathrm{~h}$ à $1 \mathrm{~h}$; o seguinte, das $13 \mathrm{~h}$ às $19 \mathrm{~h}$; o subsequente, das $7 \mathrm{~h}$ às $13 \mathrm{~h}$, encerrando o ciclo dos turnos alternantes com o horário de $1 \mathrm{~h}$ às $7 \mathrm{~h}$. No dia seguinte, após 36 horas de descanso, o que caracteriza a folga, o trabalhador reinicia o ciclo de turnos, das $19 \mathrm{~h}$ à $1 \mathrm{~h}$. Para a contagem do tempo de trabalho exercido em turno alternante (em meses) foi considerada a data de admissão do trabalhador.

Um inquérito sociodemográfico, fechado, para obtenção de dados sobre idade, escolaridade e condições de saúde dos participantes, foi elaborado e aplicado pelos pesquisadores por meio de entrevista. A utilização de álcool foi avaliada pelo AUDIT ${ }^{12}$.

Para a avaliação da QV utilizou-se The Medical Outcomes Study 36-Item Short-Form Health Survery (SF-36). A versão utilizada foi a validada em português ${ }^{13}$. O instrumento é composto por 36 itens e contempla 8 domínios: capacidade funcional, aspectos físicos, dor, estado geral de saúde, vitalidade, aspectos sociais, aspectos emocionais e saúde mental. Cada domínio recebe um escore que varia de 0 a 100 ; quanto maior o escore, melhor a percepção sobre QV. O questionário é autoaplicável, cabendo aos pesquisadores apenas observar se alguma questão deixou de ser respondida, o que ocasiona a sua invalidação. O cálculo dos escores do SF-36 foi feito de acordo com os seguintes passos ${ }^{14}$ : cálculo de cada domínio e soma dos pontos obtidos em cada item relativo ao domínio correspondente, para cada indivíduo; uso dos valores mínimos e máximos possíveis em cada item para calcular o valor transformado, com emprego na Equação 1:

$$
Y_{i j}=\frac{X_{i j}-\min _{j}}{\operatorname{má}_{j}-\min _{j}} \times 100
$$

em que: $\mathrm{i}=1,2,3, \ldots 87$ (índice do trabalhador); $\mathrm{j}$ = capacidade funcional, aspectos físicos... (cada um dos domínios); Yij = valor transformado do trabalhador i, domínio j; Xij = valor do domínio j, do trabalhador $\mathrm{i}$; mínj = valor mínimo possível para o domínio j; máxj $=$ valor máximo possível para o domínio j.

Após a entrevista e o preenchimento do SF-36, foi realizada aferição dos dados antropométricos e de composição corporal.

Os fatores de risco para doenças cardiovasculares foram definidos de acordo com a proposta da International Diabetes Federation 2007 para a população sul-americana ${ }^{7}$. Esses fatores foram a circunferência da cintura $\geq 90 \mathrm{~cm}$, triglicerídeos $\geq 150 \mathrm{mg} /$ dL, HDL colesterol $<40 \mathrm{mg} / \mathrm{dL}$, pressão arterial sistólica $\geq 130 \mathrm{mmHg}$ e/ou pressão arterial diastólica $\geq 85 \mathrm{mmHg}$ e/ou tratamento com anti-hipertensivo, LDL colesterol $\geq 160 \mathrm{mg} / \mathrm{dL}$, tabagismo, consumo de bebidas alcoólicas ${ }^{12}$ e baixo nível de atividade física. A atividade física foi avaliada pelo instrumento Internacional Physical Activity Questionnaire (Ipaq) versão 8 (forma longa), e baixo nível foi considerado para aqueles com nível de atividade física $<600$ Measure Energy Total (MET) minutos/semana ${ }^{10}$.

Para a coleta de dados antropométricos (peso, estatura e CC), os avaliados permaneciam em posição ortostática, com roupas leves e descalços. O peso foi verificado por meio do monitor de composição corporal Inbody $720^{\hat{a}}$. A estatura foi aferida pelo estadiômetro digital HM-210D, com o participante de braços estendidos ao longo do corpo, calcanhares juntos, tocando a haste vertical do estadiômetro e sem adornos na cabeça ${ }^{13}$. A partir desses dados foi calculado o IMC, sendo os critérios da OMS: baixo peso, com IMC menor que 18,5; peso adequado, entre 18,5 e 25,0; com sobrepeso entre 25,0 e 30,0; e obesidade, maior que $30,0^{3}$.

A CC foi aferida em triplicata, com fita métrica simples e inelástica, no ponto médio entre a crista ilíaca e o último arco costal. A leitura foi feita durante a expiração, com o indivíduo em pé com os braços afastados do tronco ${ }^{13}$.

A análise de composição corporal (gordura visceral, percentual de gordura corporal total e da gordura corporal por compartimento) foi realizada por meio do monitor de bioimpedância elétrica tetrapolar segmentada Inbody $720^{\hat{a}}$. Antes da avaliação da composição corporal, os participantes foram orientados, de acordo com a recomendação técnica do fabricante do instrumento, a realizar jejum absoluto de no mínimo 4 horas, a não realizar exercícios nas 12 horas anteriores, esvaziar a bexiga urinária 30 minutos antes do teste e não utilizar diurético nas 24 horas que antecederam o exame, a fim de diminuir os erros nas medidas. No momento da avaliação, os voluntários foram orientados a retirar todos 
os objetos de metal e subir descalços no monitor. Foram mensurados a gordura visceral, o percentual de gordura corporal total e o percentual de gordura corporal por compartimento.

As variáveis antropométricas e de composição corporal analisadas foram apresentadas inicialmente de forma descritiva, com valores de média e desvio-padrão para aquelas que se ajustam a uma distribuição normal (teste de Anderson-Darling), e mediana e percentis para as que não se ajustaram. Foi utilizado o teste de correlação de Spearman entre os domínios do SF-36 e as variáveis antropométricas e de composição corporal. Por meio da análise multivariada de cluster foram identificados dois grupos de variáveis semelhantes (Grupo 1 - variáveis de QV e Grupo 2 - variáveis antropométricas), que serviram de base para obtenção dos índices via técnica de componentes principais. De acordo com Kubrusly ${ }^{15}$, uma forma de obter indicadores que contenham o máximo de informação fornecida em um conjunto de variáveis selecionadas é a análise de componentes principais. Assim, em cada grupo foi aplicada essa técnica multivariada, com o intuito de construir indicadores globais para estimar os escores de cada indivíduo em cada índice, em que, por construção, as variáveis que fazem parte de cada indicador são altamente correlacionadas. Todos os pressupostos necessários para utilizar essas técnicas foram verificados.

As análises estatísticas foram efetuadas nos programas Statistical Package for Social Sciences (SPSS)
17.0, Minitab versão 15 e R (R Development Core Team 2016).

O cálculo do poder amostral (a posteriori) foi realizado utilizando o programa $\mathrm{G}^{*}$ Power versão 3.1.9.2. Para o tamanho amostral de 437 participantes utilizou-se o valor da influência da magnitude do efeito de 0,12 para as correlações; o poder estimado foi de 0,71 . Para todos os testes utilizados adotou-se o nível de significância de 5\%.

A pesquisa foi aprovada pelo Comitê de Ética em Pesquisa da Universidade Federal de Ouro Preto (CAAE: 0018.0.238.000-11).

\section{Resultados}

A Tabela 1 apresenta as características do grupo de trabalhadores estudados. Com base na mediana encontrada para o IMC $\left(26,16 \mathrm{~kg} / \mathrm{m}^{2}\right)$, conclui-se que $50 \%$ dos participantes estão na faixa de sobrepeso, além de apresentarem CC média e mediana superior a $90 \mathrm{~cm}$.

Na avaliação da QV (Tabela 2), os domínios de saúde geral e vitalidade apresentaram as menores pontuações, com medianas de 67 e 80, respectivamente.

Observa-se na Tabela 3 a correlação entre os índices antropométricos e de composição corporal e a QV.

Tabela 1 Média e mediana de idade e de indicadores de obesidade entre trabalhadores (n=437) de turnos alternantes de mineradora na região dos Inconfidentes, Minas Gerais, Brasil, 2012

\begin{tabular}{lcc}
\hline Variáveis & Média $(D P)$ & Mediana \\
\hline Idade (anos) & $35,63(6,99)$ & 34,00 \\
IMC $\left(\mathrm{kg} / \mathrm{m}^{2}\right)$ & $26,43(4,76)$ & 26,16 \\
CC $(\mathrm{cm})$ & $91,89(9,70)$ & 92,00 \\
AGV $\left(\mathrm{cm}^{2}\right)$ & $124,81(46,11)$ & 121,20 \\
\%GC & $23,36(6,94)$ & 23,40 \\
\hline
\end{tabular}

IMC - índice de massa corporal; CC - circunferência da cintura; AGV - área de gordura visceral; \%GC - percentual de gordura corporal.

Tabela 2 Pontuação mediana e percentis nos diferentes domínios da qualidade de vida entre os trabalhadores de turnos alternantes de mineradora da região dos Inconfidentes, Minas Gerais, Brasil, 2012

\begin{tabular}{lccc}
\hline \multicolumn{1}{c}{ Variáveis } & Mediana & Percentil 25 & Percentil 75 \\
\hline Aspecto físico & 100 & 100 & 100 \\
Aspecto emocional & 100 & 100 & 100 \\
Capacidade funcional & 95 & 90 & 100 \\
Dor & 84 & 72 & 100 \\
Saúde geral & 67 & 57 & 77 \\
Vitalidade & 80 & 70 & 90 \\
Aspecto social & 100 & 87,5 & 100 \\
Saúde mental & 88 & 80 & 96 \\
\hline
\end{tabular}


Tabela 3 Correlação entre os índices antropométricos, de composição corporal, tempo de trabalho e deslocamento, e a qualidade de vida de trabalhadores em turnos alternantes de mineradora da região dos Inconfidentes, Minas Gerais, Brasil, 2012

\begin{tabular}{lcccccccc}
\hline & Aspectosfísicos & $\begin{array}{c}\text { Aspectos } \\
\text { emocionais }\end{array}$ & $\begin{array}{c}\text { Capacidade } \\
\text { funcional }\end{array}$ & Dor & Saúdegeral & Vitalidade & $\begin{array}{c}\text { Aspectos } \\
\text { sociais }\end{array}$ & Saúde mental \\
\hline IMC & 0,042 & $-0,038$ & $-0,018$ & $-0,014$ & $-0,105^{*}$ & $-0,012$ & $-0,011$ & $-0,021$ \\
AGV & $-0,046$ & 0,013 & $-0,116^{*}$ & $-0,052$ & $-0,030$ & $-0,095^{*}$ & 0,016 & $-0,073$ \\
\%GC & $-0,051$ & 0,049 & $-0,100^{*}$ & 0,029 & $-0,024$ & $-0,049$ & 0,056 & $-0,019$ \\
CC & $-0,052$ & 0,019 & $-0,123^{*}$ & $-0,044$ & $-0,020$ & 0,000 & 0,028 & 0,027 \\
MGC & $-0,071$ & 0,037 & $-0,094^{*}$ & 0,002 & $-0,035$ & $-0,060$ & 0,029 & $-0,027$ \\
MGT & $-0,065$ & 0,024 & $-0,120^{*}$ & 0,001 & $-0,027$ & $-0,066$ & 0,044 & $-0,019$ \\
\%MGT & $-0,049$ & 0,023 & $-0,132^{* * *}$ & 0,010 & $-0,032$ & $-0,059$ & 0,053 & $-0,020$ \\
MGBD & $-0,062$ & 0,029 & $-0,111^{*}$ & 0,012 & $-0,027$ & $-0,052$ & 0,052 & $-0,015$ \\
\%MGBD & $-0,056$ & 0,034 & $-0,117^{*}$ & 0,024 & $-0,025$ & $-0,047$ & 0,063 & $-0,007$ \\
MGBE & $-0,063$ & 0,033 & $-0,116^{*}$ & 0,010 & $-0,031$ & $-0,062$ & 0,055 & $-0,017$ \\
\%MGBE & $-0,058$ & 0,033 & $-0,116^{*}$ & 0,022 & $-0,027$ & $-0,051$ & 0,057 & $-0,013$ \\
MGPD & $-0,070$ & 0,036 & $-0,099^{*}$ & 0,018 & $-0,020$ & $-0,041$ & 0,051 & $-0,003$ \\
\%MGPD & $-0,051$ & 0,043 & $-0,103^{*}$ & 0,033 & $-0,018$ & $-0,028$ & 0,068 & 0,006 \\
MGPE & $-0,070$ & 0,033 & $-0,098^{*}$ & 0,019 & 0,020 & $-0,045$ & 0,047 & $-0,007$ \\
\%MGPE & $-0,053$ & 0,040 & $-0,105^{*}$ & 0,035 & $-0,020$ & $-0,029$ & 0,068 & 0,005 \\
\hline
\end{tabular}

IMC - índice de massa corporal; AGV - área de gordura visceral; \%GC - percentual de gordura corporal; CC - circunferência da cintura; MGC - massa de gordura corporal; MGT - massa de gordura no tronco; \%MGT - percentual de massa de gordura no tronco; MGBD - massa de gordura no braço direito; \%MGBD percentual de massa de gordura no braço direito; MGBE - massa de gordura no braço esquerdo; \%MGBE - percentual de massa de gordura no braço esquerdo; MGPD - massa de gordura na perna direita; \%MGPD - percentual de massa de gordura na perna direita; MGPE - massa de gordura na perna esquerda; \%MGPE - massa de gordura na perna esquerda. Teste de correlação de Spearman: ${ }^{*} \mathrm{p}<0,05$ *** $\mathrm{p}<0,01$.

Deve-se ressaltar que somente a saúde geral, vitalidade e capacidade funcional se correlacionaram com os índices antropométricos e de composição corporal. O domínio de saúde geral foi correlacionado negativamente com o IMC $(\mathrm{r}=$ $-0,105 ; \mathrm{p}<0,05)$; já a vitalidade correlacionou-se com a AGV ( $r=-0,095 ; p<0,05)$. Para capacidade funcional as maiores correlações foram verificadas na CC $(r=-0,123 ; p<0,05)$ e no percentual de massa de gordura no tronco $(\mathrm{r}=-0,132 ; \mathrm{p}<0,001)$. A capacidade funcional se correlacionou negativamente com a CC $(\mathrm{r}=-0,123 ; \mathrm{p}<0,05)$. Ressalta-se que os coeficientes de correlação estimados foram significativos, mas de baixa magnitude, indicando baixa associação inversamente proporcional. A partir da análise de cluster pelo método hierárquico de Ward foram obtidos dois grandes grupos. Observou-se um único agrupamento para QV composto pelos 8 domínios do SF-36 e um segundo grupo composto por variáveis referentes a composição corporal. A variável antropométrica CC também fez parte desse grupo.

Para cada cluster encontrado utilizou-se da técnica de componentes principais para estimarmos os escores de cada indivíduo em seus respectivos indicadores, indicador de QV e de adiposidade. Aos componentes estimados foram:
Indicador de Qualidade de Vida:

$Y_{1}=0,311$ (Aspectos_Físicos) + 0,319 (Aspecto Emocional) $+0,320$ (Capacidade_Funcional) $+0,34 \overline{5}$ (Dor) + 0,312 (Saúde Geral) + 0,408 (Vitalidade) + 0,390 (Aspecto_Social) + 0,406 (Saúde_Mental)

Indicador de Adiposidade Corporal

$Z_{1}=0,195(\mathrm{AGV})+0,283(\mathrm{MGC})+0,272(\% \mathrm{GC})+$ $0,280(\mathrm{MGBD})+(\% \mathrm{MGBD})+0,279(\mathrm{MGBE})+0,281$ $(\%$ MGBE $)+0,234(\mathrm{MGT})+0,281(\% \mathrm{MGT})+0,281$ $(\mathrm{MGPD})+0.277$ (\%MGPD) + 0,276 (MGPE) + 0,273 (\%MGPE) $+0,233$ (CC)

AGV - área de gordura visceral; \%GC - percentual de gordura corporal; CC - circunferência da cintura MGC - massa de gordura corporal; MGT - massa de gordura no tronco; \%MGT - percentual de massa de gordura no tronco; MGBD - massa de gordura no braço direito; \%MGBD - percentual de massa de gordura no braço direito; MGBE - massa de gordura no braço esquerdo; \%MGBE - massa de gordura no braço esquerdo; MGPD - massa de gordura na perna direita; \%MGPD - percentual de massa de gordura na perna direita; MGPE - massa de gordura na perna esquerda; \%MGPE - percentual de massa de gordura na perna esquerda. 


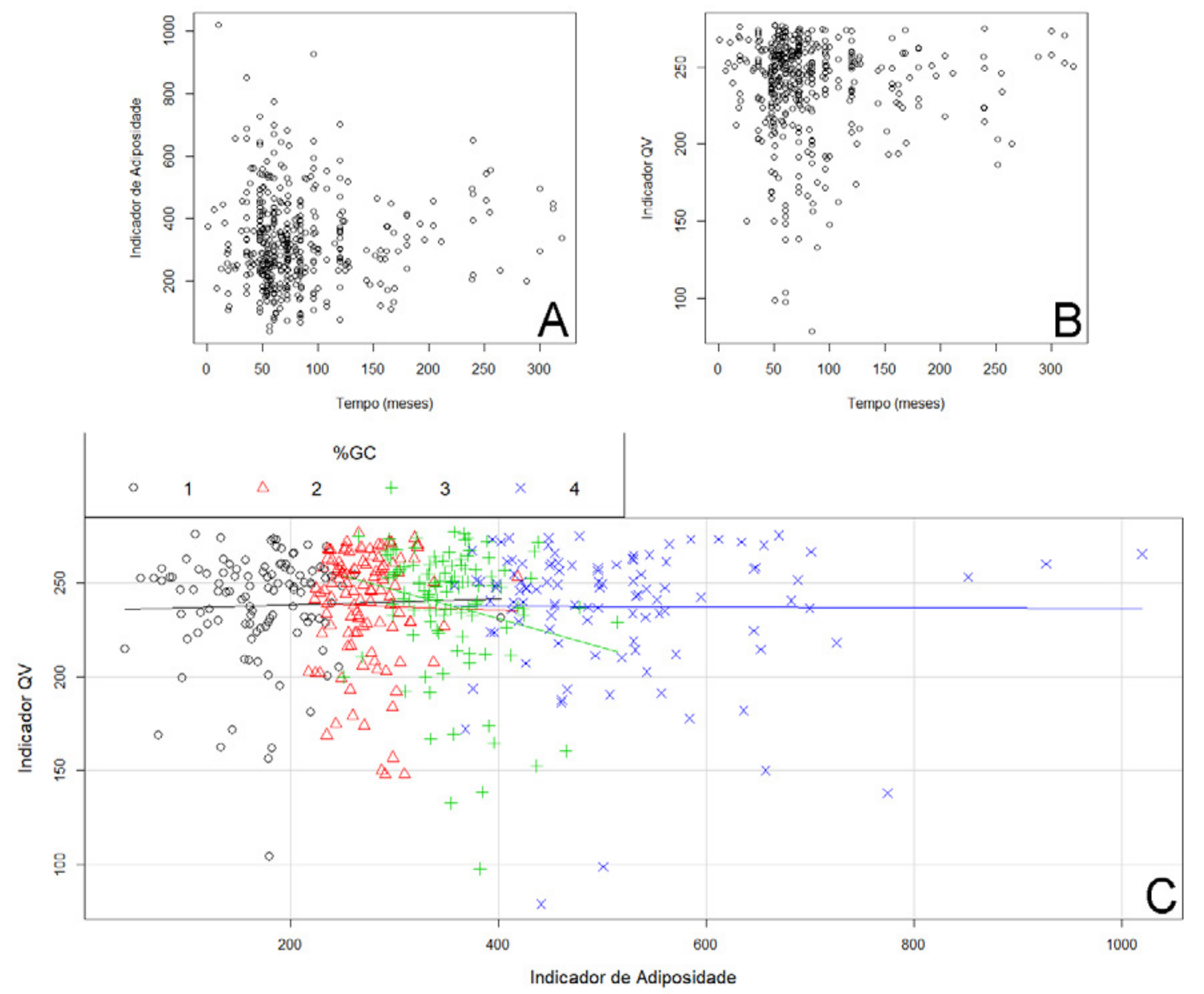

Figura 1 Escore dos trabalhadores de turnos alternantes para o indicador de Adiposidade (A) e de Qualidade de Vida (QV) (B). Escore dos trabalhadores pelos indicadores QV e Adiposidade versus quartis de porcentagem de gordura corporal (\%GC) (C)

As porcentagens de variância total explicadas pelos componentes são de $=50,4 \%$ e de $=87,1 \%$. A influência de cada fator presente nos componentes é positiva para os indicadores estimados. Ou seja, para os dois componentes, cada unidade acrescentada dos fatores para cada trabalhador gera um aumento no valor estimado dos escores.

Contudo, ao analisar os escores estimados com o tempo de trabalho de turno, não foi possível visualizar nenhuma associação entre os escores (adiposidade corporal e QV, Figura 1A e Figura 1B, respectivamente) e o tempo de trabalho em meses para cada trabalhador de turno. No entanto, observou-se maior variabilidade entre os escores encontrados para o indicador de adiposidade corporal versus tempo de trabalho em meses de atividade ocupacional em turno. Para o indicador QV esta variabilidade também é visualizada, mas em menor magnitude.

Na análise em 3 dimensões do indicador de adiposidade corporal, por meio da variável \%GC, observa-se que aqueles indivíduos com alto percentual de gordura corporal apresentam maior variabilidade para o indicador QV e maiores escores para o indicador de adiposidade (Figura 1C). Os valores da variável \%GC estão ordenados e apresentados pelos seus quartis.

\section{Discussão}

Neste estudo a capacidade funcional apresentou correlação negativa com os índices de composição corporal e CC, e o domínio saúde geral e vitalidade com o IMC e AGV, respectivamente, ou seja, uma relação inversamente proporcional. Um aumento em tais índices pode representar diminuição da QV nesses domínios. No entanto, não foi observada correlação entre os índices antropométricos e de composição corporal com os domínios aspectos físicos, emocionais, sociais e saúde mental da QV. Estes resultados são respaldados por outros estudos quanto ao domínio saúde mental. Huang et al. ${ }^{16}$ e Yanbo et al. ${ }^{3}$, mostraram que o domínio saúde mental não se associa ao excesso de peso. Outro fator relevante a ser considerado é a idade: a população 
estudada é de adultos jovens, e alguns estudos mostram a influência da idade na percepção da QV entre indivíduos mais velhos, mas provavelmente devido a doenças $^{17}$. Ressalta-se que pesquisas são necessárias para elucidar os mecanismos pelos quais o excesso de peso afeta domínios específicos da QV. Destaca-se também que este estudo analisou dados transversais, o que possibilita avaliar somente a QV atual dos trabalhadores.

As maiores pontuações obtidas nos domínios da QV foram para os aspectos físico, emocional, social e capacidade funcional, resultados estes semelhantes ao estudo de Wu et al. ${ }^{18}$.

Edimansyah et al. ${ }^{19}$ estudaram 728 trabalhadores de montagem de automóveis na Malásia e mostraram que $64,9 \%$ relataram "boa" ou "muito boa" QV usando o WHOQOL-BREF. Os autores concluíram que uma possível explicação para os achados seria a presença das novas tecnologias e processos de trabalho capazes de tornar o processo operacional mais prático. No entanto, ressalta-se que outros fatores podem estar envolvidos na boa percepção da QV pelos trabalhadores. Höfelmann e Blank ${ }^{20}$ observaram que trabalhadores industriais do sexo masculino, economicamente ativos e em sua maioria jovens tiveram baixa prevalência de autoavaliação de saúde negativa.

Outra explicação para a QV declarada seria a ocorrência do "efeito do trabalhador sadio", no qual haveria uma seleção progressiva de pessoas, com a exclusão e demissão daquelas em piores condições de saúde, ficando remanescentes aquelas com a saúde mais preservada.

Durante um período de 8 anos, Froom et al. ${ }^{21}$ acompanharam uma coorte de 5.547 trabalhadores industriais do sexo masculino israelenses e observaram o efeito do trabalhador sadio nessa população, que apresentou menor risco de mortalidade por doenças cardiovasculares e câncer.

Metzner e Fischer ${ }^{22}$ realizaram um estudo transversal com 43 trabalhadores de turnos diurnos e noturnos, e observaram que os últimos desenvolveram estratégias de enfrentamento eficazes a esta atividade laboral, tais como acompanhamento clínico, psicológico e adaptações sociais com envolvimento da família. Estratégias de enfrentamento ou de coping são esforços cognitivos e comportamentais para lidar com situações de dano, ameaça ou desafio quando há mudanças na rotina. Apenas esforços conscientes e intencionais são considerados estratégias de coping. Enfatizamos que a população deste estudo contava com serviço de assistência à saúde, passando por exames periódicos.

O domínio saúde geral, seguido por vitalidade e dor, foram aqueles com menores pontuações. Resultados semelhantes foram encontrados por Martinez e
Latorre ${ }^{23}$ com trabalhadores do setor elétrico do estado de São Paulo, assim como Cruz et al. ${ }^{24}$, em pesquisa com 755 indivíduos da população geral de Porto Alegre.

Kheiraoui et al. ${ }^{25}$, ao avaliarem a QV de profissionais de saúde, observaram que as maiores pontuações para o domínio de saúde mental foram daqueles que passaram mais tempo inseridos em atividades sociais diurnas (com a família e amigos). Wong et $\mathrm{al}^{26}$, ao avaliaram a QV em diferentes profissionais motoristas, observaram que o hábito de fazer exercícios regulares e a realização das refeições em família tiveram melhores escores para o componente mental. Ressalta-se que o grupo de trabalhadores avaliados neste estudo, na maioria dos domínios, apresentou escores maiores que em outros estudos, como a pontuação mediana de 88 para o domínio saúde mental, fato que talvez possa ser explicado pelo pouco tempo trabalhando em alternância de turnos, o que poderia diminuir possíveis efeitos na cognição, particularmente na atenção e concentração. Marquié et al. ${ }^{27}$ associaram o declínio das funções cerebrais ao tempo em que o indivíduo trabalha em regime de turnos.

A presença de obesidade em trabalhadores de turnos tem sido relatada por diversos estudos ${ }^{5,8,9}$. Pesquisa realizada com 475 trabalhadores de indústria em Joinville detectou prevalência de $53 \%$ de excesso de peso diagnosticado pelo $\mathrm{IMC}^{28}$. Neste estudo foi observado que as médias dos índices nutricionais (IMC, CC, \%GC e AGV) estavam acima dos valores de normalidade para a população, indicando uma tendência ao excesso de adiposidade corporal.

Investigações sobre a relação entre QV e IMC têm demonstrado que o decréscimo da QV se acentua com o aumento de peso ${ }^{3,4}$, assim como o excesso de gordura corporal está relacionado a uma pior $\mathrm{QV}^{5}$.

Contudo, ao correlacionar os critérios de QV com os parâmetros antropométricos e de composição corporal verificou-se que a capacidade funcional foi inversamente associada a todos os índices de composição corporais e à CC, exceto com ao IMC, o que indica que o decréscimo da capacidade funcional pode estar ligado ao aumento desses índices. Esses resultados corroboram os encontrados por Fachineto ${ }^{29}$ em uma população de trabalhadores rurais de Santa Catarina.

O IMC foi correlacionado apenas com o domínio saúde geral. Em Taiwan, homens obesos tiveram pior percepção de sua saúde geral em comparação aos de peso normal ou sobrepeso ${ }^{16}$. É importante ressaltar que o IMC se torna um parâmetro limitado, pois não é capaz de detectar a proporção de massa livre de gordura e de gordura corporal, ou seja, os trabalhadores podem apresentar um IMC dentro do padrão ideal e, no entanto, possuírem uma quantidade de gordura corporal acima do ideal; ou apresentarem um IMC acima do recomendado e possuírem uma 
quantidade de gordura corporal ideal ${ }^{26,28}$. Assim, outros métodos mais precisos, como a aferição da composição corporal, devem ser considerados.

Neste estudo, a análise de composição corporal realizada por meio da bioimpedância tetrapolar segmentada, permitiu estimar melhor a gordura corporal dos trabalhadores. Verificou-se que a AGV foi inversamente associada à capacidade funcional e vitalidade. Segundo Rosmond e Bjorntorp ${ }^{30}$, o desenvolvimento de obesidade abdominal está associado à diminuição de saúde autorrelatada e baixa autoestima, provavelmente pela limitação da realização de tarefas básicas diárias, prejudicando assim a QV. Ressalta-se que por serem motoristas, que exercem suas atividades sentados, com os comandos próximos às mãos e, portanto, com pouca mobilidade, o excesso de gordura corporal na região abdominal poderia também dificultar o desenvolvimento de suas atividades de trabalho, prejudicando o desempenho, a produtividade e, portanto, sua QV.

Embora não tenha sido encontrada correlação entre os domínios aspecto físico, dor e os índices antropométricos e de composição corporal, esses não devem ser de menor importância, já que evidências apontam os prejuízos do excesso de peso sobre os tais domínios. Estudo realizado em Portugal com 203 trabalhadores verificou que aqueles com excesso de peso ou obesidade estavam mais propensos a ter dor músculo-esquelética em comparação àqueles com peso normal ${ }^{31}$. Além disso, dores nos membros inferiores são mais frequentes entre os homens com IMC $\geq 25 \mathrm{~kg} / \mathrm{m}^{2}$, provavelmente porque o elevado peso corporal coloca tensões nos ossos e articulações ${ }^{28}$. Considerando que estes trabalhadores são motoristas de caminhões fora de estrada, uma boa condição física é fundamental, devido ao desgaste provocado pela atividade laboral.

Os índices antropométricos e de composição corporal também não se correlacionaram com os aspectos emocionais, sociais e saúde mental. Outro estudo não verificou impactos negativos do excesso de peso sobre os componentes mentais da QV, como saúde mental, aspectos funcionais, sociais e vitalidade ${ }^{24}$.

Os resultados observados neste estudo, como a falta de relação entre os indicadores antropométricos e composição com aspectos mentais, sociais e saúde mental, podem ser decorrentes do sistema de rotação de turno rápida, definição dos dias de descanso e a manutenção do sistema de mudança de turno regu$\operatorname{lar}^{32}$. Como os resultados de estudos multicêntricos demonstram, trabalhar mais de 40 horas por semana está associado a eventos adversos ${ }^{32}$. Em longo prazo, o trabalho em turnos pode resultar em interações entre vários fatores de risco nos diferentes domínios da $\mathrm{QV}$, as quais podem ter impactos com diferentes percepções entre os trabalhadores ${ }^{33}$.
Quanto ao resultado da análise de cluster, a CC e as variáveis de composição corporal foram incluídas no mesmo grupo, o que sugere que para avaliar o estado nutricional ambas podem ser consideradas, assim também permitiram a construção de um indicador de adiposidade corporal. Os 8 domínios do SF-36 foram agregados em um único cluster, definindo assim um indicador de QV e reafirmando seu conceito multidimensional (abrangendo aspectos físicos, sociais e emocionais). Esse resultado reforça a ideia de que o SF-36 é um instrumento de fácil aplicação capaz de medir a QV da população geral e com eficácia comprovada em amostras de todo mundo ${ }^{22}$.

No Brasil, estudos sobre QV foram centrados principalmente em portadores de doenças ou em profissionais da área da saúde, no entanto, trabalhadores do contexto industrial são pouco avaliados, principalmente os que exercem suas atividades em turnos alternantes em mineradoras ${ }^{14,21,23}$. Os dados apresentados salientam a dificuldade de comparar a QV e o excesso de peso em relação a outros grupos de trabalhadores brasileiros. Por outro lado, tais resultados expressam a importância de que novas pesquisas sejam feitas com essa população, a fim de estudar o uso de medidas intervencionistas no estilo de vida e no campo da saúde do trabalhador, a fim de contribuir para a melhoria da QV destes trabalhadores.

Além da limitação de um estudo transversal, o que impossibilitou avaliar a capacidade preditiva dos indicadores, destacamos o autopreenchimento do instrumento de QV. Contudo, podem ser destacados alguns pontos que favorecem a validade deste estudo, como medidas aferidas por técnicas apropriadas e realizadas por pesquisadores treinados.

\section{Conclusão}

A pontuação de alguns domínios da QV, tais como saúde geral, vitalidade e principalmente capacidade funcional, apresentou diminuição com o aumento da adiposidade corporal, e os indicadores de composição corporal e antropométrico (CC) se correlacionaram com a capacidade funcional nos trabalhadores de turno alternantes. Já o IMC apresentou correlação negativa com a saúde geral. A AGV foi o único índice correlacionado à vitalidade, o que pode contribuir para o aumento da variabilidade da QV nestes indivíduos. No entanto, observou-se que a QV e os indicadores de adiposidade corporal não se associaram ao tempo que o indivíduo trabalha em regime de turno. Assim, recomenda-se a realização de estudos longitudinais que permitam observar se a QV é influenciada pelo tempo de serviço nesses trabalhadores de turno da mineração. 


\section{Contribuições de autoria}

Todos os autores contribuíram substancialmente para delinear o estudo, levantar, analisar e interpretar os dados, bem como para a elaboração e aprovação da versão final do manuscrito.

\section{Referências}

1. World Health Organization. Programme on mental health. WHOQOL user manual. Genebra: WHO; 1998 [citado em 2019 fev 7]. Disponível em: https:/apps.who.int/iris/bitstream/ handle/10665/77932/WHO_HIS_HSI_Rev.2012.03_ eng.pdf? sequence $=1$ \&isAllowe $\overline{\mathrm{d}}=\mathrm{y} \& \overline{\mathrm{u}} \mathrm{u}=1$

2. Sampaio JR. Qualidade de vida no trabalho: perspectivas e desafios atuais. Psicologia: Organizações e Trabalho. 2012;12(1):121-136.

3. Zhu Y, Wang Q, Pang G, Lin L, Origasa H, Wang $\mathrm{Y}$, et al. Association between body mass index and health-related quality of life: the "Obesity Paradox" in 21,218 adults of the Chinese General Population. PLoS One. 2015;10(6):e0130613.

4. Zwaan M, Petersen I, Kaerber M, Burgmer R, Nolting B, Legenbauer T, et al. Obesity and quality of life: a controlled study of normalweight and obese individuals. Psychosomatics. 2009;50(5):474-82.

5. Akinpelo A, Akinola OT, Gbiri CA. Adiposity and quality of life: a case study from a urban center Nigeria. J Nutr Educ Behav. 2009;41(5):347-52.

6. Esquirol Y, Perret B, Ruidavets JB, Marquie JC, Dienne E, Niezborala M, et al. Shift work and cardiovascular risk factors: new knowledge from the past decade. Arch Cardiovasc Dis. 2011;104(12):636-68.

7. 7. Kivimäki M, David Batty G, Hublin C. Shift work as a risk factor for future type 2 diabetes: evidence, mechanisms, implications, and future research directions. Plos Med. 2011;8(12):1-3.

8. Morgan PJ, Collins CE, Plotnikoff RC, Cook AT, Berthon B, Mitchell S, et al. Efficacy of a workplace-based weight loss program for overweight male shift workers: the workplace POWER (Preventing Obesity Without Eating like a Rabbit) randomized controlled trial. Prev Med. 2011;52(5):317-25.

9. Pepłońska B, Burdelak W, Krysicka J, Buowska A, Marcinkiewicz A, Sobala W, et. al. Night shift work and modifiable lifestyle factors. Int J Occup Med Environ Health. 2014;27(5):693-706.

10. Codarin MAF, Moulatlet EM, Nehme P, Ulhôa M, Moreno CRC. Associação entre prática de atividade física, escolaridade e perfil alimentar de motoristas de caminhão. Soc Saude. 2010;19(2):418-28.

11. Battaus MRB, Monteiro MI. Perfil sociodemográfico e estilo de vida de trabalhadores de uma indústria metalúrgica Rev Bras Enferm. 2013;66(1):52-8.

12. Moretti-Pires RO, Corradi-Webster CM. Adaptação e validação do Alcohol Use Disorder Identification
Test (AUDIT) para população ribeirinha do interior da Amazônia, Brasil. Cad Saúde Pública. 2011;27(3):497-509.

13. Ciconelli RM, Ferraz MB, Santos W, Meinão I, Quaresma MR. Tradução para a língua portuguesa e validação do questionário genérico de avaliação de qualidade de vida SF-36 (Brasil SF-36). Rev Bras Reumatol. 1999;39(3):143-150.

14. 14. Pimenta FAP, Fabrícia FS, Henrique OGT, Carlos FSA, Camila FR, Thaissa OC, et al. Avaliação da qualidade de vida de aposentados com a utilização do questionário SF-36. Rev Assoc Med Bras. 2008;54(1):55-60.

15. Kubrusly, L. S. Um procedimento para calcular índices a partir de uma base de dados multivariados. Pesquisa Operacional. 2001;21(1):107-117.

16. Huang IC, Frangakis C, Wu A. The relationship of excess body weight and health related quality of life: evidence from a population study in Taiwan. Int J Obes. 2006;30(8):1250-9.

17. Nascimento CM, Ribeiro AQ, Cotta RMM, Acurcio FA, Peixoto SV, Priore SE, et al. Factors associated with functional ability in Brazilian elderly. Arch Gerontol Geriatr. 2012;54(2):89-94.

18. Wu SY, Li HY, Tian J, Zhu W, Li J, Wang XR. Health-related quality of life and its main related factors among nurses in China. Ind Health. 2011;49(2):158-65.

19. Edimansyah BA, Rusli BN, Naing L, Mohamed Rusli BA, Winn T. Relationship of psychosocial work factors and health-related quality of life in male automotive assembly workers in Malaysia. Ind Health. 2007;45(3):437-48.

20. Höfelmann DA, Blank N. Auto-avaliação de saúde entre trabalhadores de uma indústria no sul do Brasil. Rev Saúde Pública. 2007;41(5):777-87.

21. Froom P, Melamed S, Kristal-Boneh E, Benbassat J, Ribak J. Healthy volunteer effect in industrial workers. J Clin Epidemiol. 1999;52(8):731-5.

22. Metzner RJ, Fischer FM. Fadiga e capacidade para o trabalho em turnos fixos de doze horas. Rev Saúde Pública. 2001;35(6):548-53.

23. Martinez MC, Latorre MRDO. Fatores associados à capacidade para o trabalho de trabalhadores do Setor Elétrico. Cad Saúde Pública. 2009;25(4):761-72.

24. Cruz LN, Fleck MPA, Oliveira MR, Camey SA, Hoffmann JF, Bagattini AM, et al. Healthrelated quality of life in Brazil: normative data for the SF-36 in a general population sample 
in the south of the country. Cien Saude Colet. 2013;18(7):1911-21.

25. Kheiraoui F, Gualano MR, Mannocci A, Boccia A, La Torre G. Quality of life among healthcare workers: a multicentre cross-sectional study in Italy. Public Health. 2012;126(7):624-29.

26. Wong CK, Fung CS , Siu SC, Wong KW, Lee $\mathrm{KF}$, Lo YY, et al. The impact of work nature, lifestyle, and obesity on health-related quality of life in Chinese professional drivers. J Occup Environ Med. 2012;54(8):989-94.

27. Marquié JC, Tucker P, Folkard S, Gentil C, Ansiau D. Chronic effects of shift work on cognition: findings from the VISAT longitudinal study. Occup Environ Med. 2015;72(4):258-64

28. Höfelmann DA, Blank N. Excesso de peso entre trabalhadores de uma indústria: prevalência e fatores associados. Rev Bras Epidemiol. 2009;12(4):657-70.
29. Fachineto S, Sampaio TV, Cavassini I, Camini J. Avaliação da adiposidade corporal e qualidade de vida de trabalhadores rurais do gênero masculino de São Miguel do Oeste/SC. Cinergis. 2011;12(1):40-7.

30. Rosmond R, Bjorntorp P. Quality of life, overweight, and body fat distribution in middleaged men. Behav Med. 2000;26(2):90-4.

31. Moreira-Silva I, Santos R, Abreu S, Mota J. Associations between body mass index and musculoskeletal pain and related symptoms in different body regions among workers. Sage Open. 2013;3(2):1-6.

32. Costa G. Shift work and health: current problems and preventive actions. Saf Health Work. 2010;1(2):112-123.

33. Dall'Ora C, Ball J, Recio-Saucedo A, Griffiths P. Characteristics of shift work and their impact on employee performance and wellbeing: a literature review. Int J Nurs Stud. 2016;57:12-27. 